\title{
IDENTIFICATION OF MOST EFFECTIVE FORM OF PULSE VOLTAGE SUPPLY OF ELECTRIC WINDINGS OF ACOUSTIC MAGNETIC DEVICE PROCESSING LIQUID IN WATER PIPES
}

\author{
Alexey Korzhakov, Sergei Oskin \\ Kuban State Agrarian University, Russia \\ korzhakovalex@gmail.com, mechanization@kubsau.ru
}

\begin{abstract}
There is a situation when industrial enterprises are forced to spend huge amounts of money to fight with hard deposits (scale) occurring in cooling systems and thermal-power devices on the surface of heating or cooling. The resulting scale reduces the heat transfer, a reduction in the cross-section of the pipes, or a decrease in the period of exploitation and productivity of the equipment used. A new method of non-reagent treatment, based on the combination of acoustic and rotating magnetic fields in the working zone, is proposed. The new method will be called acousto-magnetic. By means of acousto-magnetic treatment, it is possible to slow the formation of inorganic deposits significantly, to reduce the rate of internal corrosion, to remove the film of fungi and bacteria from the inner surface of pipelines, without interruptions in the system, without interference in the operation of the system, without reagents and harm to the environment. One of the main factors affecting the efficiency of the device is the form of a pulsed supply voltage. The results of the investigation of the influence of the impulse voltage on the change of the voltage gradient of the magnetic field in the working zone of the acoustic and magnetic device (AMD) model are presented in the article. To conduct the research, a hypothesis is advanced about the possibility of increasing the efficiency of the acoustic and magnetic field of the device by means of various forms of impulse voltage. For the experimental study of the hypothesis, an AMD model was developed, using the ELCUT 6.1 software complex. In the process of modeling, a geometric model was created, then the physical properties of the model blocks were set, and spectral analysis of the signals was conducted using the MATLAB software application package. The obtained results show the possibility of increasing the efficiency of AMD based on the pulse voltage of different forms.
\end{abstract}

Keywords: modeling, ELCUT, alternating magnetic field, acoustic and magnetic device, method of finite elements, spectral analysis, MATLAB.

\section{Introduction}

There is a situation when industrial enterprises are forced to spend huge amounts of money to fight with hard deposits (scale) occurring in cooling systems and thermal-power devices on the surface of heating or cooling. The resulting scale reduces the heat transfer, a reduction in the cross-section of the pipes, or a decrease in the period of exploitation and productivity of the equipment used.

Cleaning of equipment and pipes from scale is a time-consuming and expensive process, involving the violation of the equipment operation, with the purchase and use of chemical reagents. Geothermal energy needs a complete replacement of all pipes after each heating season.

Traditionally, mechanical and chemical cleaning is used to remove deposits. Arsenal of means is very wide; each technology has its advantages and disadvantages. But almost all methods are aimed at eliminating the problems that have arisen, rather than at preventing their occurrence.

Currently, for descaling in the production process devices of magnetic treatment are used. In fact, they are permanent magnets or electro-magnets. They are relatively simple in design and relatively inexpensive, but their main disadvantages include the impossibility of a flexible regulation of the output parameter values, resulting in liquid media with different properties. One way to solve the existing problem is to introduce non-reagent methods of cleaning various systems from scale [1].

A new method of non-reagent treatment, based on the combination of acoustic and rotating magnetic fields in the working zone, is proposed. The new method will be called acousto-magnetic [2]. By means of acousto-magnetic treatment it is possible to slow the formation of inorganic deposits significantly, to reduce the rate of internal corrosion, to remove the film of fungi and bacteria from the inner surface of pipelines, without interruptions in the system, without interference in the operation of the system, without reagents and harm to the environment. Devices of appropriate power are mounted over the pipeline.

The implementation of the method is based on an acoustic magnetic device, which has obtained several patents [3-5]. The acoustic magnetic device can be used in various industries, including heat power engineering. An important factor is the efficiency of liquid treatment. 
One of the main factors affecting the efficiency of the device is the form of a pulsed supply voltage. The design parameters of the device are established in the process of modelling in the ELCUT and MATLAB environments.

\section{Materials and methods}

To understand the problem, we will describe a new method of reagentless water treatment, based on the combination of acoustic and rotating magnetic fields in the working zone. The method of reagentless water treatment is carried out as follows. Ultrasonic acoustic vibrations and rotating oppositely directed electromagnetic fields are used as a combined physical impact on the flow of water. To create acoustic and rotating electromagnetic fields, magnetic circuits of the system of ferrite rings with electric windings are used, which are supplied with a three-phase alternating voltage in the resonant ultrasonic frequency range of $32-35 \mathrm{kHz}$.

To implement the method, a device is used comprising a cylindrical non-magnetic body that has a magnetostrictive source of ultrasonic vibrations inside, and an electromagnetic system consisting of the magnetic circuit and windings with leads for connection to a power source outside. In the electromagnetic system, the magnetic circuit is in the form of a ferrite ring.

When applying a three-phase alternating voltage to the windings of the ferrite ring, a rotating electromagnetic field is created, concentrating in the annular gap, where the stream of water flows. A certain configuration of the coils allows the device to make the maximum use of the electromagnetic energy of the winding, which creates rotating magnetic and acoustic fields. The frequency of the electric current is selected so as to create a resonance effect that forces the ferrite core to compress and decompress, reproducing the sound vibrations acting on the liquid. This method of water treatment promotes the stability of water more than any other known methods.

Modeling of the electromagnetic field in the ELCUT environment involves the development of the equivalent circuit for the electromagnetic system of the acoustic magnetic device.

The electrical part of the equivalent circuit for the electromagnetic system of the acoustic magnetic device consists of three electrical circuits having active resistances $R_{1}, R_{2}, R_{3}$, inductances $L_{1}$, $L_{2}, L_{3}$ and mutual inductances $M_{12}, M_{13}, M_{23}$.

The first circuit has the parameters $R_{1}, L_{1}, i_{1}$ and is switched on to the pulse voltage $U_{1}$; the second circuit $R_{2}, L_{2}, i_{2}$ is switched on to the impulse voltage $U_{2}$; the third circuit $R_{3}, L_{3}, i_{3}$ is switched on the impulse voltage $U_{3}$. In general, the circuit is a system of three subsystems (circuits $1,2,3$ ). Let us write down a system of equations describing the voltage drop on each circuit.

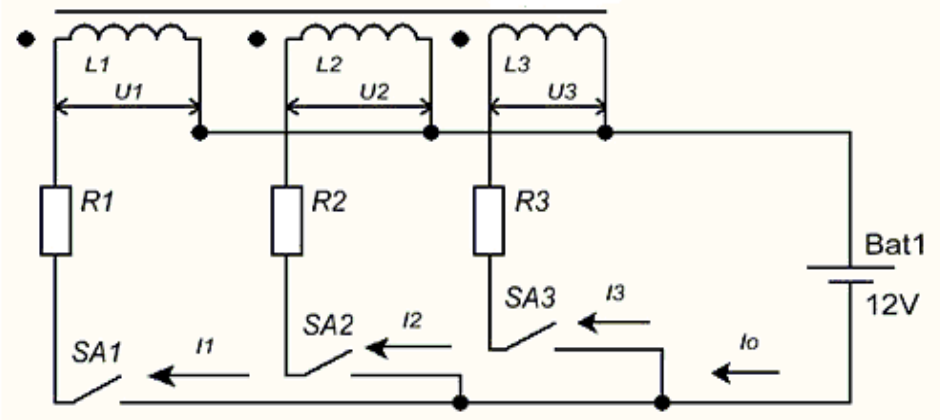

Fig. 1. Equivalent circuit of electromagnetic component of acousticmagnetic device

The total EMF induced in three circuits at the moment of switching on SA1, SA2 and SA3 switches:

$$
\left\{\begin{array}{c}
e_{1}=i_{1} R_{1}+\frac{d}{d t}\left(L_{1} i_{1}+M_{12} i_{2}+M_{13} i_{3}\right) \\
e_{2}=i_{2} R_{2}+\frac{d}{d t}\left(L_{2} i_{2}+M_{21} i_{1}+M_{23} i_{3}\right) \\
e_{3}=i_{3} R_{3}+\frac{d}{d t}\left(L_{3} i_{3}+M_{31} i_{1}+M_{32} i_{2}\right)
\end{array}\right.
$$


where $e_{1}-$ total EMF induced in the first circuit,

$e_{2}-$ total EMF induced in the second circuit,

$e_{3}-$ total EMF induced in the third circuit.

The first step is to create a problem such as the "Magnetic field of alternating currents" in the ELCUT 6.1 environment. Having established the properties of the problem, we proceed to the construction of the geometric model of the device [6]. The diagram of the device is presented in a section for a more graphic representation of the magnetic field pattern. Fig. 2 shows a geometric model in the ELCUT 6.1 environment.

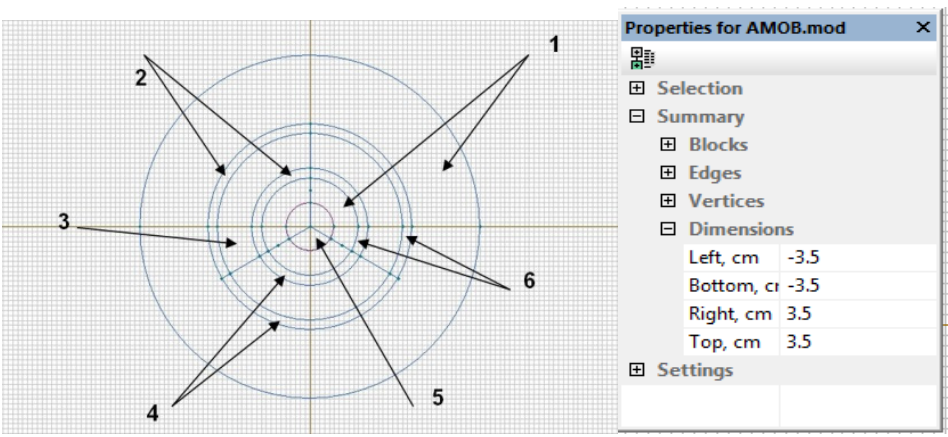

Fig. 2. Geometric model in ELCUT 6.1 environment: 1 - body of the device; 2 - first winding; 3 - ferrite ring; 4 - second winding; 5 - working zone; 6 - third winding

Following the creation of the geometric model of the problem, the physical properties of objects, as well as the boundary conditions, are specified with the help of marks. One winding is $120^{\circ}$ from the circle of the ferrite ring. The frequency at which the device is simulated is $18 \mathrm{kHz}$. The choice of precisely this frequency is due to the fact that at a given frequency the ferrite ring enters the resonance [7]. Then an electrical circuit is created to power the device.

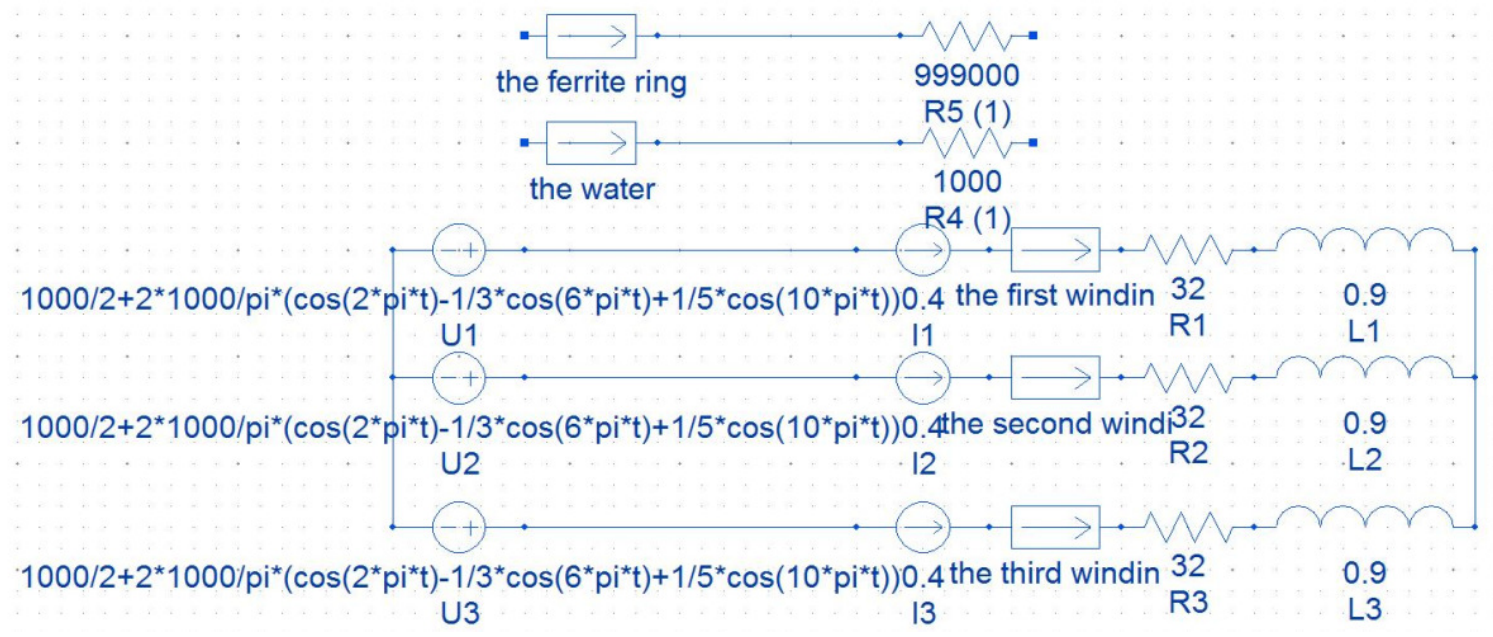

Fig. 3. Electrical circuit in environment of ELCUT 6.1 signal form of square waves

Three pulse voltages of various shapes were fed as power: saw-shaped voltage, triangular voltage and meander.

Voltage of the meander type: $u(t)=\frac{e}{2}+\frac{2 e}{\pi}\left(\cos \omega t-\frac{1}{3} \cos 3 \omega t+\frac{1}{5} \cos 5 \omega t \ldots\right)$.

Triangular voltage: $u(t)=\frac{2 e}{\pi^{2}}\left(\cos \omega t-\frac{1}{9} \cos 3 \omega t+\frac{1}{25} \cos 5 \omega t \ldots\right)$.

Saw-shaped voltage: $u(t)=\frac{2 e}{\pi}\left(\sin \omega t-\frac{1}{2} \sin 2 \omega t+\frac{1}{3} \sin 3 \omega t-\frac{1}{4} \sin 4 \omega t \ldots\right)$, 
where $\mathrm{e}$ - voltage amplitude,

$$
\omega=\frac{2 \pi}{T}-\text { circular frequency of the main harmonic [8]. }
$$

Next, a finite element mesh is constructed in all the used parts of the geometric model, with a discretization step of 0.5 . The geometric model allows specifying physical properties of objects and boundary conditions [9].

Fig. 4 shows the magnetic field pattern, when voltage of the meander type is applied to the device, but Fig. 5 shows the pattern of the magnetic field, when triangular voltage is applied to the device. Fig. 6 gives distribution of maximum intensity depending on phase.

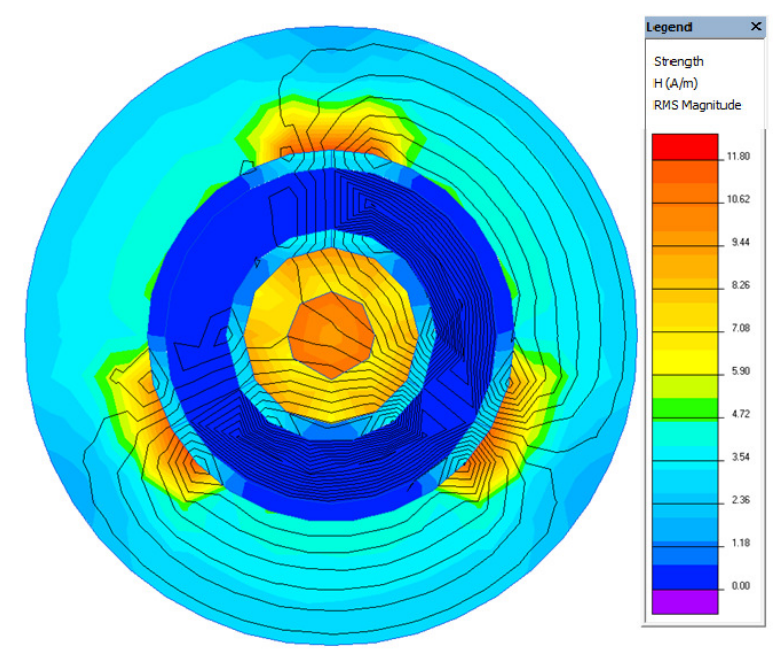

Fig. 4. Distribution of maximum tension when applying voltage of meander type

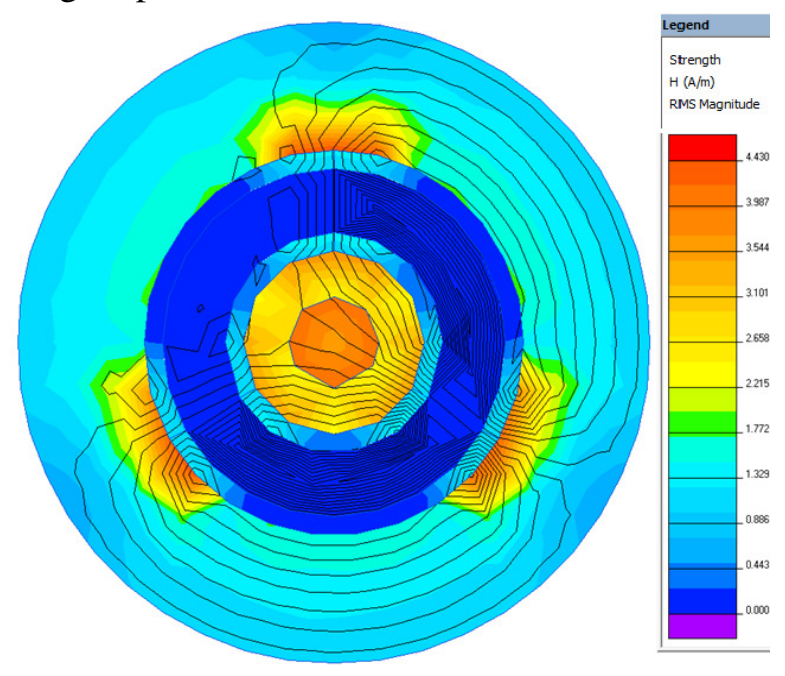

Fig. 5. Distribution of maximum tension when applying triangular voltage
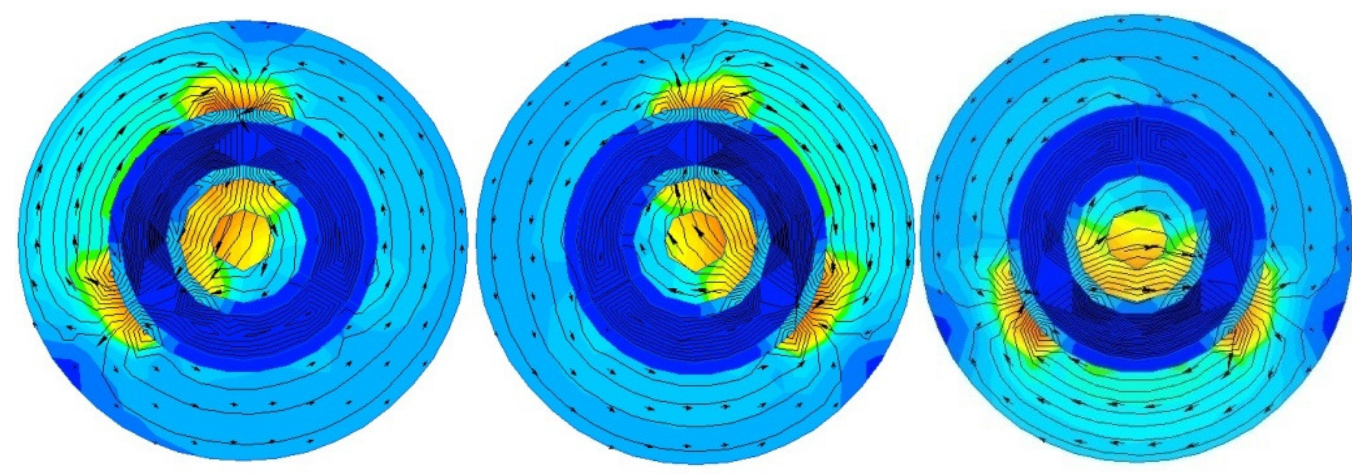

Fig. 6. Distribution of maximum intensity depending on phase

The analysis of the obtained results made it possible to construct a table of maximum/minimum tension in the working zone of the acoustic-magnetic device model, depending on the shape of the voltage pulse signal. The results are shown in Table 1.

Based on the results of modeling, the maximum intensity of the magnetic field in the working area of the acoustic magnetic device model is achieved, when the signal of the meander form is used as the supply voltage. The acoustic effect of waveforms on preventing deposits in piping was not evaluated, because it was not included in the subject of the study.

To determine the effectiveness of the pulse signal, a spectral analysis was made. The main criterion for comparing the signals was the amplitude of harmonics in the operating frequency range of the device. The analysis was carried out in the package of MATLAB applied programs [9]. 
Magnetic field intensity in the working area of the acoustic magnetic device model depending on the form of the pulse signal

\begin{tabular}{|c|c|c|}
\hline Forms of the signal & $\begin{array}{c}\text { Maximum magnetic } \\
\text { field intensity, } \mathbf{A} \cdot \mathbf{m}^{-\mathbf{1}}\end{array}$ & $\begin{array}{c}\text { Minimum magnetic field } \\
\text { intensity, } \mathbf{A} \cdot \mathbf{m}^{-\mathbf{1}}\end{array}$ \\
\hline Meander & 11.8 & $109 \cdot 10^{-5}$ \\
\hline Triangular voltage & 4.43 & $41 \cdot 10^{-5}$ \\
\hline
\end{tabular}

\section{Results and discussion}

On the basis of theoretical studies and modeling, a model of the device was made and its testing confirmed the theoretical studies. The influence of the shape of the impulse voltage of the acoustic magnetic device on the anti-scale effect was investigated at the experimental stand described in the article [10]. The studies confirmed the maximum efficiency of the application of the pulse voltage supply of the acoustic magnetic device of the "meander" form.

The experimental data obtained closely coincide with the model constructed. An industrial sample of an acoustic magnetic device is shown in Fig. 7.

Production tests confirmed the efficiency of the device. Fig. 8 shows a section of the pipe that was rejected in the result of routine maintenance work performed after the heating season.

The efficiency of the device was tested during the year of its operation. As a result of routine maintenance it was found that the amount of deposits on the pipes began to tend to zero. Fig. 9 shows a damper that has been used throughout the heating season.

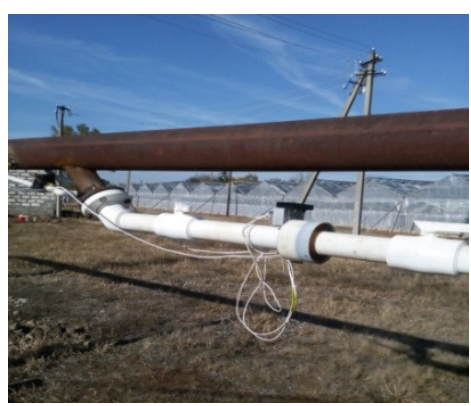

Fig. 7. Appearance of geothermal water supply pipe and device with electronic components installed on it

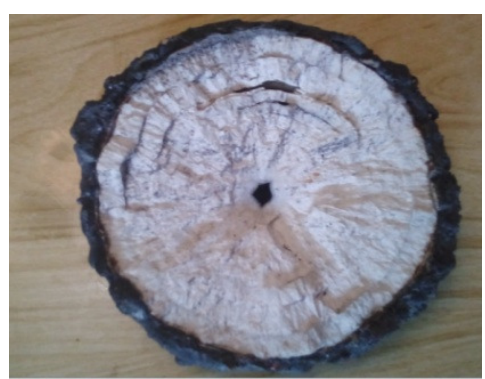

Fig. 8. Pipe section with tsalt deposits before installation of device

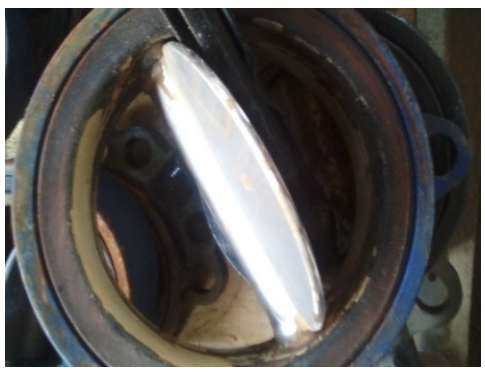

Fig. 9. Damper in disassembled form after heating season with device

Before installing the device, the dampers were discarded after two weeks of operation. Traditionally, a magnetic device is used to treatment of water and solution. The device parameters are:

- conditional diameter (mm): 80; 100; 200; 600;

- nominal pressure (MPa): 1.6;

- capacity of processed water $\left(\mathrm{m}^{3} \cdot \mathrm{h}^{-1}\right): 25-600$;

- magnetic field strength $\left(\mathrm{kA} \cdot \mathrm{m}^{-1}\right): 200$;

- consumed power by electromagnet (kW): 0.35-1.80; 
- dimensions of magnet (mm): $260 \times 420 ; 440 \times 835 ; 520 \times 950 ; 755 \times 1100$;

- weight of magnet $(\mathrm{kg}): 40 ; 200 ; 330 ; 1000$.

Ten of these devices are installed on a pipe with a diameter of $80 \mathrm{~mm}$ demonstrate power consumption $3.5 \mathrm{~kW}$, weight $-400 \mathrm{~kg}$. With the installation of ten acoustic and magnetic devices (Fig. 6), the power consumption is 70-100 watts, weight $-8 \mathrm{~kg}$. However, the performance for the processed water is the same $\left(25 \mathrm{~m}^{3} \cdot \mathrm{h}^{-1}\right)$. The measured value of the magnetic field strength of the acoustic and magnetic device in the working area is $15 \mathrm{~A} \cdot \mathrm{m}^{-1}$ with $\mathrm{RMS}=1$. It is planned to optimize the overall dimensions and power of the device.

\section{Conclusions}

1. Comparison of the amplitudes of the harmonics of the signals on the graphics clearly shows that the largest part of the energy is concentrated in the first harmonic, and the maximum amplitude of the voltage applied to the windings is achieved in the form of a meander, unlike other forms of impulse voltage. Accordingly, the excited current in the windings of the device and the intensity of the magnetic field with this form of the signal reach the highest values.

2. The gradient of the magnetic field intensity is concentrated in the working zone of the device. The maximum effective form of the pulse voltage supply for the acoustic magnetic device is the meander, which is confirmed by the experimental studies at the stand with a closed circuit and production tests.

\section{References}

[1] Tarasik V.P. Mathematical modeling of technical systems. - Mn.: DesignPro, 2004, 640 p.

[2] Korzhakov A.V. Analysis of mathematical equations describing the acoustic and electromagnetic fields of the acoustic magnetic device, and establishing the similarity criteria for the creation of new constructions of acoustic magnetic devices designed for for use in hydroponic installations / Korzhakov A.V., Loiko V.I., Korzhakova S.A., Korzhakov V.E. // Polythematical electronic scientific journal of the Kuban State Agrarian University (Scientific journal of KubSAU), Krasnodar: KubSAU, 2016. - No. 03 (117). - IDA: 1171603031. [online][15.01.2017] Available at: http: //ej.kubagro.ru/2016/03/pdf/31.pdf.

[3] Korzhakov A.V., Korzhakov V.E., Kirillov N.P. Device for reagentless water treatment // Author's certificates for inventions BI No. 13, 1992. Author's certificate No. 1724594.

[4] Korzhakov V.E., Kramarenko B.D., Pleshakov V.V., Korzhakov A.V. Device for the non-reagent treatment of liquids // Author's certificates for inventions BI No. 38, 1989. Author's certificate No. 1514726.

[5] Korzhakov A.V., Korzhakov V.E., Oskin S.V. A device for protection against the formation of deposits on the surfaces of pipelines of the heat supply systems // Patent of the Russian Federation No. 2635591, C23F 15/00 49/06 / Application 2017100470. 09.01.2017. Publ. on 11/14/2017. Bul. No. 32.

[6] Antonov S.N. Devices of magnetic water treatment. Designing, modeling and research: a monograph / S.N. Antonov, A.I. Adoshev, I.K. Sharipov, V.N. Shemyakin.- Stavropol: AGRUS, 2014 .-220 p.: illustr. -Bibliography: pp. 206-216.

[7] Agranat B.A. Fundamentals of Physics and Technology of Ultrasound: A manual for higher schools. / Agranat B.A., Dubrovin M.N., Khavsky N.N. - M.: Vyssh. shk., 1987, 352 p.

[8] Tebenikhin E.F.Reagentless methods of water treatment in power plants. - M., Energoizdat, 1985.

[9] Korzhakov A.V. Analysis of the existing hypotheses of the mechanisms of the effects of acoustic and magnetic fields on the treated liquid / Korzhakov A.V., Oskin S.V., Loiko V.I., Korzhakova S.A., Korzhakov V.E. // Polythematical electronic scientific journal of the Kuban State Agrarian University (Scientific journal of KubSAU) [Electronic resource]. - Krasnodar: Number: 130 (06), June 2017 Published on: 30.06.2017, IDA: 1301706017. [online][15.01.2017] Available at: http://ej.kubagro.ru/2017/06/pdf/17.pdf.

[10] Korzhakov V.E. Study of the efficiency of the acoustic magnetic treatment of water systems // Proceedings of the FORA, No. 2, 1997. - Maikop: ASU, [online][15.01.2017] Available at: http://fora.adygnet.ru/files/43/16-1997.pdf. 